\title{
LHC data and cosmic ray coplanarity at superhigh energies
}

\author{
R.A. Mukhamedshin ${ }^{\text {a }}$ \\ Institute for Nuclear Research, Russian Academy of Sciences, Moscow, Russia
}

\begin{abstract}
A new phenomenological model FANSY 2.0 is designed, which makes it possible to simulate hadron interactions via traditional and coplanar generation of most energetic particles as well as to reproduce a lot of LHC (ALICE, ATLAS, CMS, TOTEM, LHCf) data. Features of the model are compared with LHC data. Problems of coplanarity are considered and a testing experiment is proposed.
\end{abstract}

\section{Introduction}

A number of models of hadron interactions with nuclei is concurrently applied in cosmic-ray experiments as none of them can explain the entire experimental data set of EAS features. Besides, a number of phenomena observed in mountain-based and stratospheric X-rayemulsion chamber (XREC) experiments are not yet explained. One of these interesting phenomena is the so-called coplanarity of the most energetic cores of $\gamma$-ray-hadron families, i.e., groups of high-energy ( $E \gtrsim$ $n \cdot 1 \mathrm{TeV}$ ) particles in relatively young EAS cores initiated by protons and nuclei of the primary cosmic radiation (PCR) (see Sect. 3).

The phenomenological model FANSY 1.0 was designed a few years ago, which helped to understand general features of coplanar events [1]. FANSY 1.0 gives reasonable results as compared with experimental data on intensity of $\gamma$-ray families, energy dependence of the muon and hadron spectra and so on [1].

However, FANSY 1.0 cannot properly reproduce LHCf data on high- $X_{F} \gamma$-rays e.g. [2], and neutrons, which are really important for cosmic ray experiments, as well as the transversal size of $\gamma$-ray families, e.g. [3].

To improve this situation, a new phenomenological model FANSY 2.0 is designed, which simulates traditional and coplanar particle generation and reproduce experimental data with a higher accuracy as compared with FANSY 1.0. FANSY 2.0 reproduces a number of ALICE, ATLAS, CMS, TOTEM data on charged, strange and charmed stable and resonance particle generation, LHCf data on high- $\mathrm{X}_{F}$ gamma-rays and neutrons.

This paper is devoted to $p p$ interactions simulated at superhigh energies $(\sqrt{s}=900 \mathrm{GeV}-13 \mathrm{TeV})$. Compatibility of FANSY 2.0 and LHC data will be discussed in more detail in another work [4].

This paper is organized as follows. Section 2 presents comparison of LHC data and simulated results. Coplanarity problem is briefly considered in Sect. 3. Final comments are given in Conclusion.

\section{High energy interactions}

\subsection{Cross sections}

Figure 1 shows FANSY 2.0's results (curve) and data of different experiments on the energy dependence of the inelastic $p p$ cross section $\sigma_{\text {inel }}^{p p}$ (top); single and double diffraction sections, $\sigma_{S D}^{p p}$ and $\sigma_{D D}^{p p}$ (bottom). Inelastic $p p$ cross section data include results of the ATLAS [5] and CMS [6] Collaborations at $\sqrt{s}=13 \mathrm{TeV}$.

\subsection{Low-(pseudo)rapidity data}

Experimental LHC data are mainly related to low(pseudo)rapidity particles. This kinematic range is not very important for consideration of cosmic-ray-initiated EAS's in the atmosphere. However, models, which cannot describe these data, will inevitably cause mistrust. Experimental and simulated data are shown in Sect. 2 with filled and empty symbols, respectively.

\subsubsection{Charged particles}

Figure 2 shows ATLAS $d N_{c h} / d \eta$ distributions of charged particles at $-2.1<\eta<2.1, \quad n_{c h} \geq 2$, and $p_{t}>0.1 \mathrm{GeV} / \mathrm{c}$ in NSD $p p$ interactions at $\sqrt{s}=13 \mathrm{TeV}$ (circles) [7], $7 \mathrm{TeV}$ (squares) and $900 \mathrm{GeV}$ (diamonds) [8].

Figure 3 shows experimental $d N_{c h} / d \eta$ distributions of charged particles in $p p$ NSD interactions at $\sqrt{s}=7 \mathrm{TeV}$ of the CMS experiment [9] at $-2.5<\eta<2.5$ (left) and the LHCb experiment at $2.0<\eta<4.5$ (right) [10].

\subsubsection{Strange particles}

Figure 4 shows FANSY 2.0 and CMS $d N / d|y|$ distributions of $\mathrm{K}_{s}^{0}$ mesons in $p p$ NSD interactions at $\sqrt{s}=900 \mathrm{GeV}$ (lower squares) and $\sqrt{s}=7 \mathrm{TeV}$ (upper triangles) [11].

Figure 5 shows FANSY 2.0 and LHCb experiment's $d \sigma / d y$ distributions of $\phi$ mesons with $0.6<p_{t}<$ $5.0 \mathrm{GeV} / \mathrm{c}$ at $\sqrt{s}=7 \mathrm{TeV}$ (upper squares) [12].

\subsubsection{Charmed particles}

Among different aims, FANSY 2.0 is designed to study the "forward-physics" aspects of the generation of charmed

a e-mail: rauf_m@mail.ru

(c) The Authors, published by EDP Sciences. This is an Open Access article distributed under the terms of the Creative Commons Attribution License 4.0 (http://creativecommons.org/licenses/by/4.0/). 


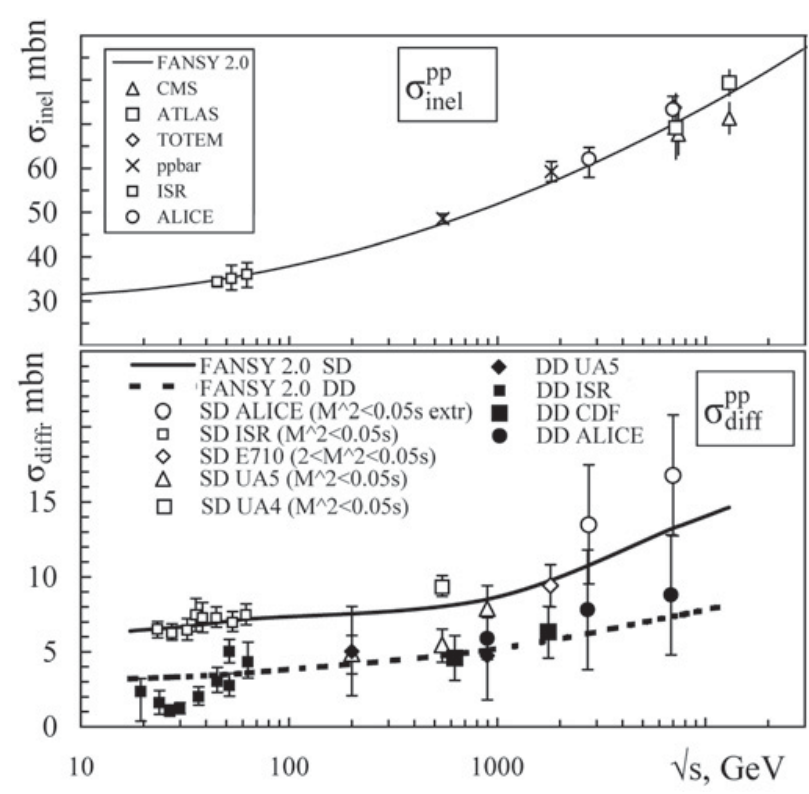

Figure 1. Experimental (points) and FANSY 2.0's (curve) energy dependencies of inelastic $p p$ cross section (top); single and double diffraction sections (bottom).

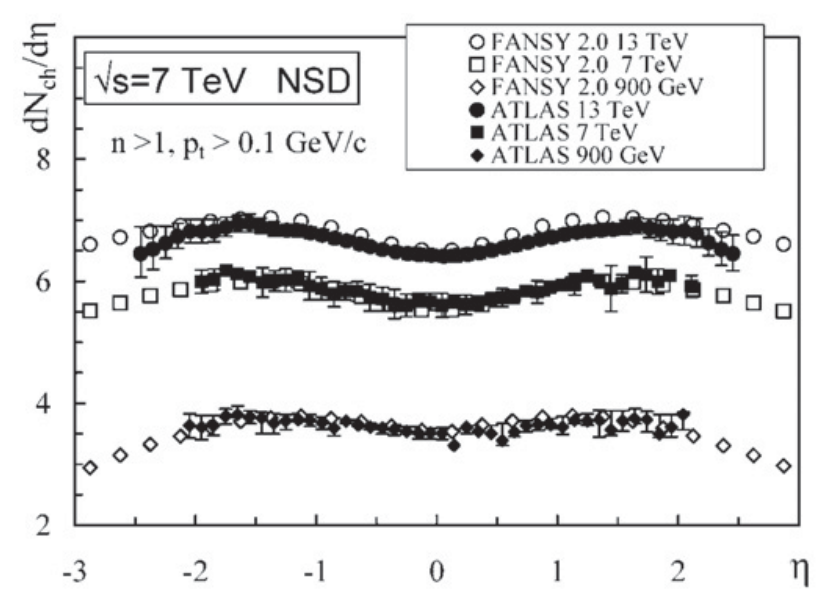

Figure 2. FANSY 2.0 and ATLAS $d N_{c h} / d \eta$ distributions of charged particles at $-2.1<\eta<2.1, n_{c h} \geq 2$, and $p_{t}>$ $0.1 \mathrm{GeV} / \mathrm{c}$ in NSD $p p$ interactions at $\sqrt{s}=13 \mathrm{TeV}$ (circles), $7 \mathrm{TeV}$ (squares) and $900 \mathrm{GeV}$ (diamonds).

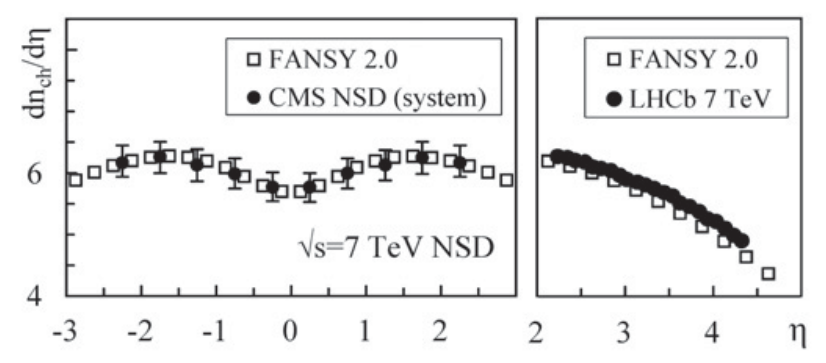

Figure 3. FANSY 2.0 and experimental $d N_{c h} / d \eta$ distributions of charged particles in $p p$ NSD interactions at $\sqrt{s}=7 \mathrm{TeV}$ of the CMS experiment at $-2.5<\eta<2.5$ (left) and LHCb experiment at $2.0<\eta<4.5$ (right).

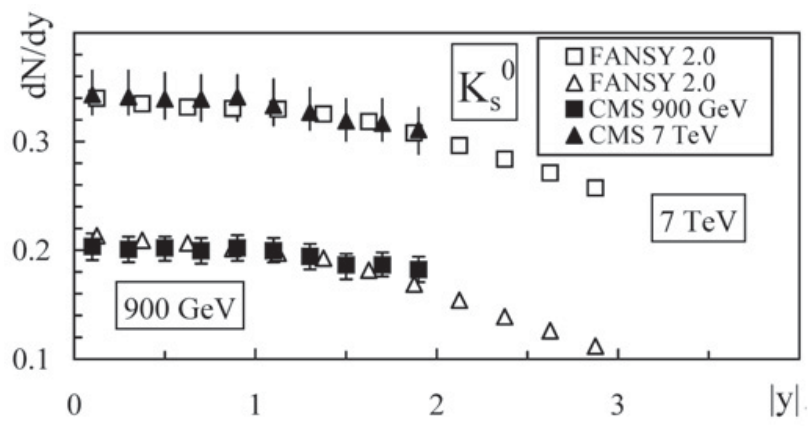

Figure 4. CMS $d N / d|y|$ distributions of $\mathrm{K}_{s}^{0}$ mesons in $p p$ NSD interactions at $\sqrt{s}=900 \mathrm{GeV}$ (lower squares) and $\sqrt{s}=7 \mathrm{TeV}$ (upper triangles).

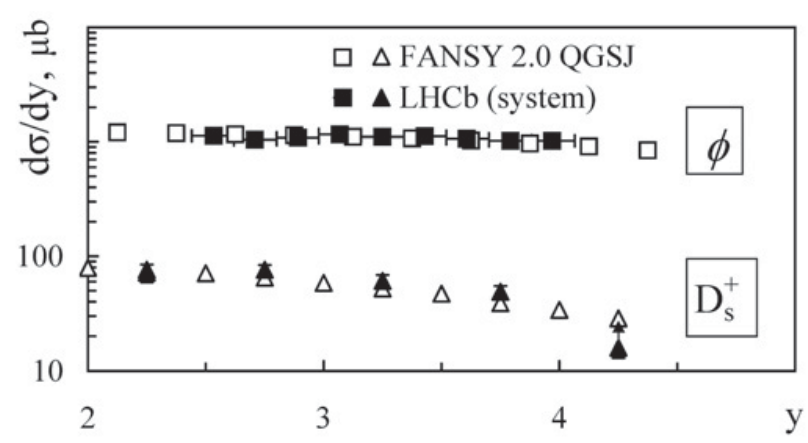

Figure 5. FANSY 2.0 and $\mathrm{LHCb} d \sigma / d y$ distributions of $\phi$ mesons at $0.6<p_{t}<5.0 \mathrm{GeV} / \mathrm{c}$ (upper squares); $\mathrm{D}_{s}^{ \pm}$mesons at $1.0<p_{t}<8.0 \mathrm{GeV} / \mathrm{c}$ (lower triangles) at $\sqrt{s}=7 \mathrm{TeV}$.

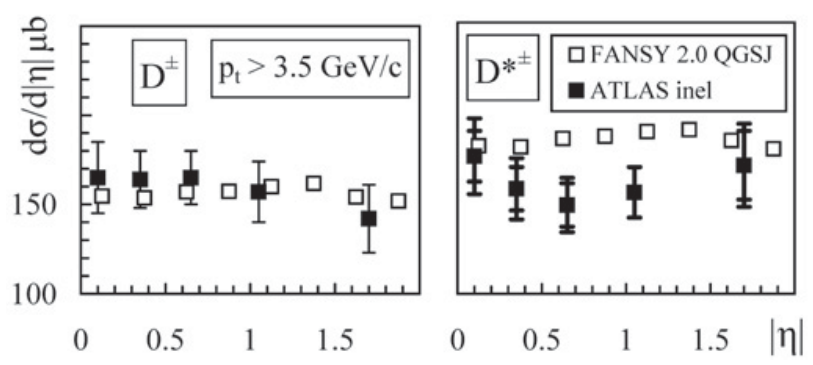

Figure 6. ATLAS and FANSY 2.0's $d \sigma / d|\eta|$ distribution of $\mathrm{D}^{ \pm}$ mesons (left) and $\mathrm{D}^{* \pm}$ mesons (right) with $p_{t}>3.5 \mathrm{GeV} / \mathrm{c}$.

particles. So these particles are considered below in more detail as compared with light-quark hadrons.

Figure 5 shows $\mathrm{LHCb} d \sigma / d y$ distribution of $\mathrm{D}_{s}^{ \pm}$ mesons with $1.0<p_{t}<8.0 \mathrm{GeV} / \mathrm{c}$ at $\sqrt{s}=7 \mathrm{TeV}$ in $p p$ interactions (lower triangles).

Figure 6 shows FANSY 2.0 and ATLAS $d \sigma / d|\eta|$ distributions of $\mathrm{D}^{ \pm}$(left) and $\mathrm{D}^{ \pm \pm}$(right) mesons with $p_{t}>3.5 \mathrm{GeV} / \mathrm{c}$ in $p p$ interactions at $\sqrt{s}=7 \mathrm{TeV}$ [13].

Figure 7 shows ALICE $d \sigma / d p_{t}$ distributions of $\mathrm{D}^{+}$ (top,left), $\mathrm{D}^{0}$ (top,right) mesons as well as strange $\mathrm{D}_{s}^{+}$(bottom,left), vector $\mathrm{D}^{*+}$ (bottom,right) mesons at $|y|<0.5$ and $\sqrt{s}=7 \mathrm{TeV}$ in $p p$ interactions [14]. Experimental systematic uncertainties are approximately equal to statistical ones.

Figure 8 shows LHCb data on $d^{2} \sigma / d p_{t} d y$ distributions of $\mathrm{D}^{+}$mesons in five rapidity regions, 


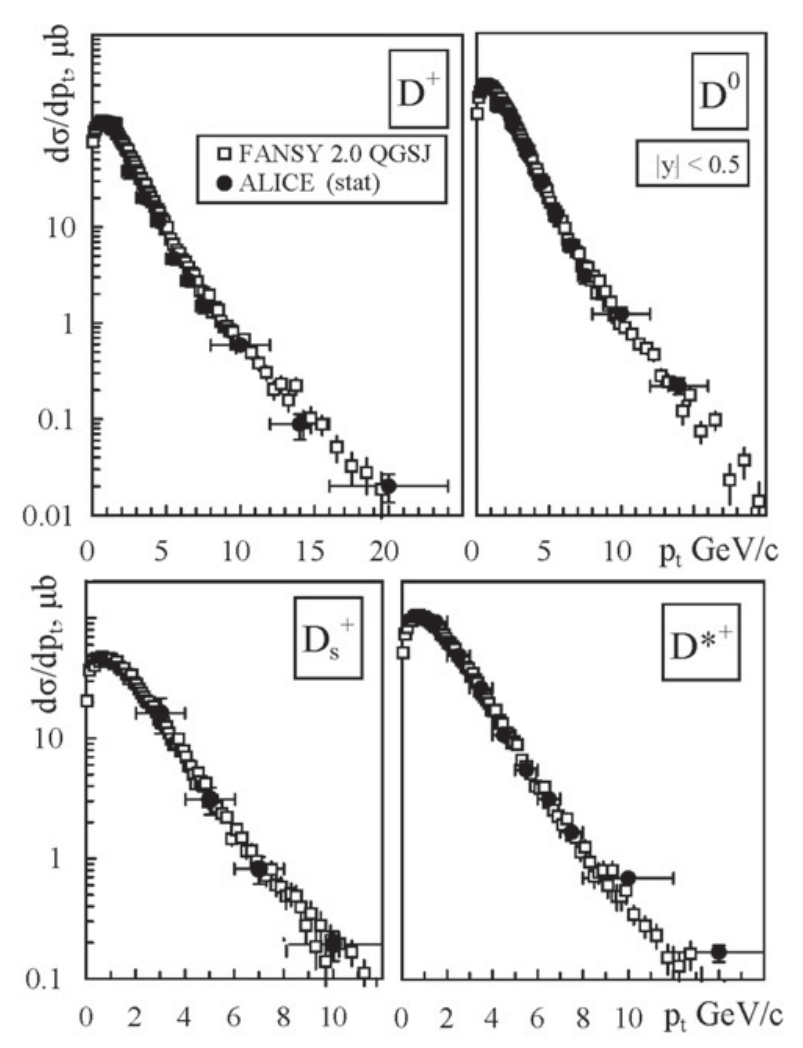

Figure 7. ALICE $d \sigma / d p_{t}$ distributions of $\mathrm{D}^{+}$(top, left), $\mathrm{D}^{0}$ (top, right) mesons as well as strange $\mathrm{D}_{s}^{+}$(bottom,left), vector $\mathrm{D}^{*+}$ (bottom,right) mesons at $|y|<0.5$.

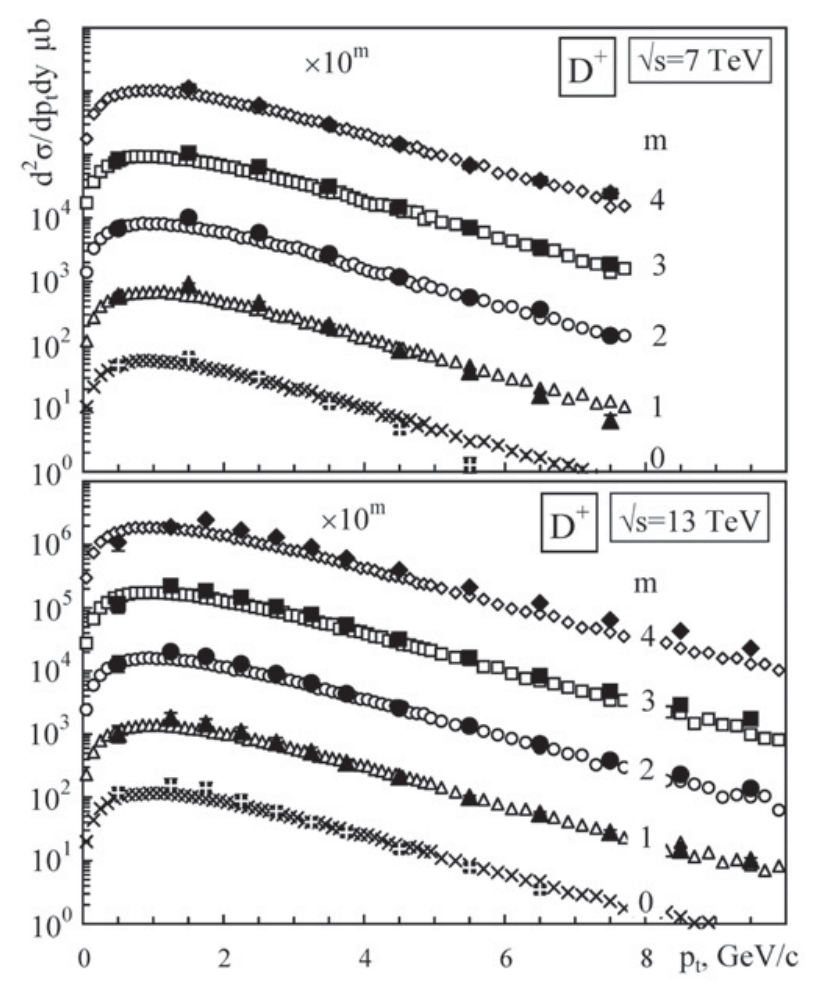

Figure 8. $\mathrm{LHCb} d^{2} \sigma / d y d p_{t}$ distributions of $\mathrm{D}^{0}$ mesons in five rapidity regions $(2.0-2.5,2.5-3.0,3.0-3.5,3.5-4.0,4.0-$ 4.5) at $\sqrt{s}=7 \mathrm{TeV}$ (top) and $\sqrt{s}=13 \mathrm{TeV}$ (bottom) with filled diamonds, squares, circles, triangles, and crosses, respectively. Spectra are multiplied by $10^{m}$, where $m=4,3,2,1,0$ for the above-mentioned $y$ ranges, respectively.

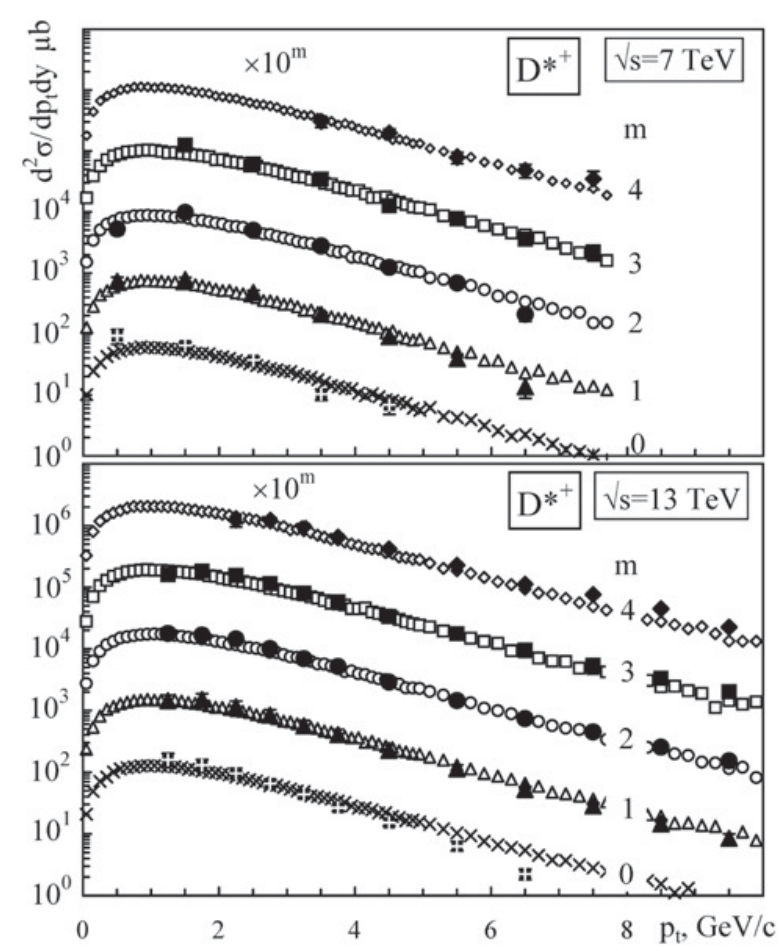

Figure 9. $\mathrm{LHCb} d^{2} \sigma / d p_{t} d y$ distributions of $\mathrm{D}^{*+}$ mesons in five rapidity regions. Notations are the same as in Fig. 8.

Table 1. $\mathrm{D}^{0}, \mathrm{D}^{+}$and $\mathrm{D}^{*+}$ charmed-meson generation cross sections (mbarn). ALICE data include statistical and systematical errors. FANSY 2.0 data include statistical errors.

\begin{tabular}{lccc}
\hline Particles & $\begin{array}{c}p_{t}, \\
\mathrm{GeV} / \mathrm{c}\end{array}$ & $\begin{array}{c}\text { ALICE } \\
\sigma \pm \text { stat. } \pm \text { syst. }\end{array}$ & $\begin{array}{c}\text { FANSY 2.0 } \\
\sigma \pm \text { stat. }\end{array}$ \\
\hline $\mathrm{D}^{0}$ & $1-16$ & $412 \pm 33_{-140}^{+55}$ & $457 \pm 6$ \\
$\mathrm{D}^{+}$ & $1-24$ & $198 \pm 24_{-73}^{+42}$ & $201 \pm 4$ \\
$\mathrm{D}^{*+}$ & $1-24$ & $203 \pm 23_{-67}^{+30}$ & $180 \pm 2$ \\
\hline
\end{tabular}

namely, at $2.0-2.5,2.5-3.0,3.0-3.5,3.5-4.0$, and $4.0-4.5$ at $\sqrt{s}=7 \mathrm{TeV}$ (top) and $\sqrt{s}=13 \mathrm{TeV}$ (bottom) with diamonds, squares, circles, triangles, and crosses, respectively $[15,16]$. Spectra are multiplied by $10^{m}$, where $m=4,3,2,1,0$ for the above-mentioned rapidity ranges, respectively.

Figures 9 and 10 show LHCb $d^{2} \sigma / d p_{t} d y$ distributions of $\mathrm{D}^{*+}$ and $\mathrm{D}_{s}^{+}$mesons, respectively $[15,16]$. All the notations are the same as in Fig. 8.

Figure 11 shows data of the ALICE experiment on $d^{2} \sigma / d y d p_{t}$ of $\omega^{0}$ (squares) and $\phi$ mesons (triangles) at $2.5<y<4.5$ [17]. Comparison of cross sections of generation of these mesons gives information on the suppression of strange quark generation.

Figure 12 shows experimental $d \sigma / d p_{t}$ spectra of $\mathrm{D}^{ \pm}$ (left) and $\mathrm{D}^{* \pm}$ (right) mesons at $|\eta|<2.1$ by the ATLAS experiment at $\sqrt{s}=7 \mathrm{TeV}$.

Table 1 shows ALICE and FANSY 2.0 cross sections of $\mathrm{D}^{0}, \mathrm{D}^{+}$and $\mathrm{D}^{*+}$ charmed-meson generation at $|\eta|<$ 0.5 in wide $p_{t}$ ranges.

Table 2 shows ALICE and FANSY 2.0 ratios of yields of charmed mesons, $\mathrm{D}_{s}^{+} / \mathrm{D}^{+}$and $\mathrm{D}_{s}^{+} / \mathrm{D}^{0}$, as well as a ratio of prompt vector mesons to prompt vector+pseudoscalar mesons, $P_{V}=\mathrm{D}^{*} /\left(\mathrm{D}^{*+}+\mathrm{D}^{+}\right)$, at $|\eta|<0.5$. 


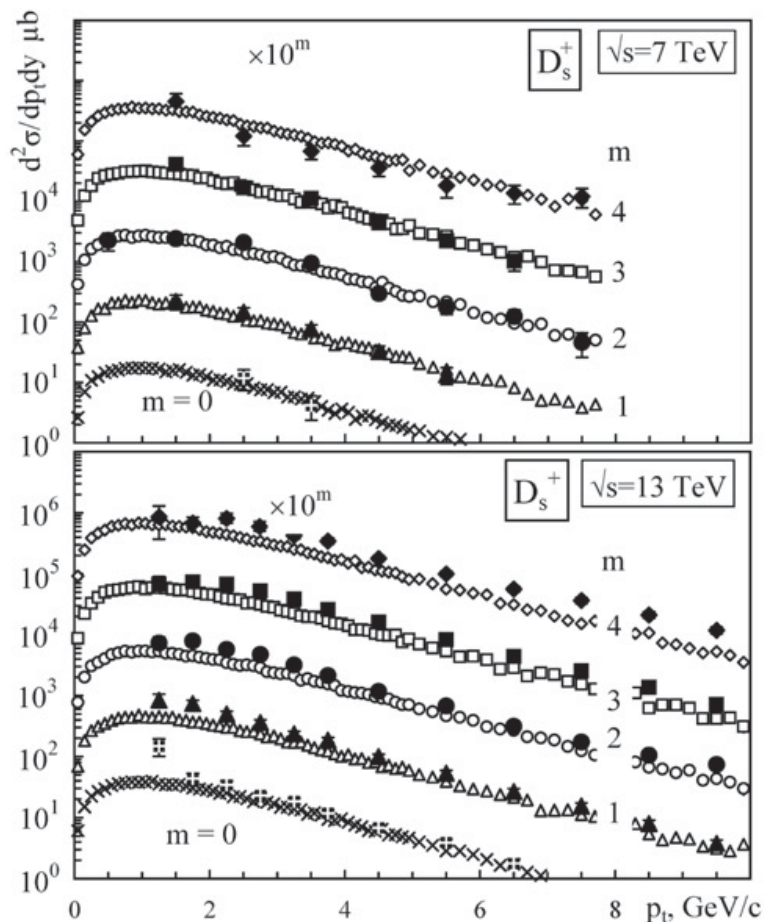

Figure 10. $\mathrm{LHCb} d^{2} \sigma / d p_{t} d y$ distributions of $\mathrm{D}_{s}^{+}$mesons in five rapidity regions. Notations are the same as in Fig. 8.

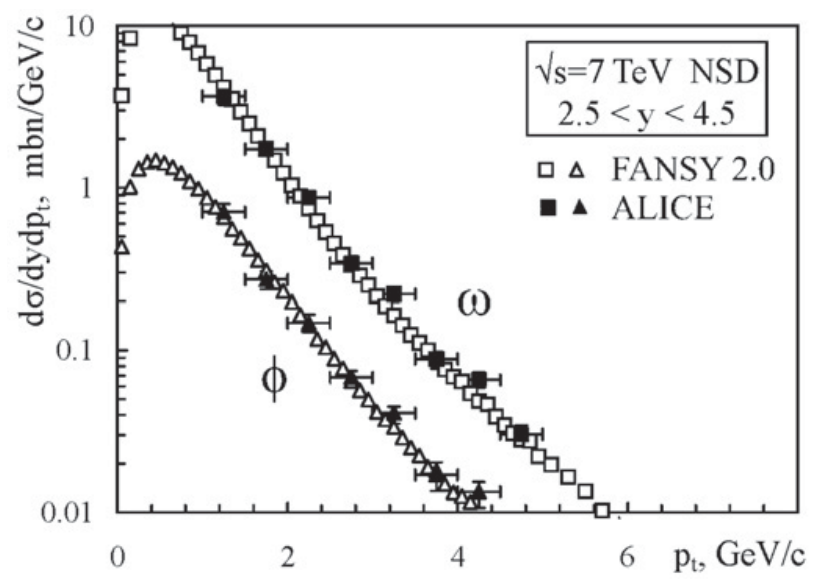

Figure 11. FANSY 2.0 and ALICE $d^{2} \sigma / d y d p_{t}$ distributions of $\omega^{0}$ (upper squares) and $\phi$ (lower triangles) mesons, respectively, at $2.5<y<4.5$ at $\sqrt{s}=7 \mathrm{TeV}$.

\subsubsection{High $\eta$ and $X_{F}$ data}

Experimental high $\eta$ and $X_{F}$ data are more interesting for cosmic ray experiments. Unfortunately, at the present time the specific design of colliders makes it possible to derive highest $\eta$ and $X_{F}$ data for low- $p_{t}$ neutral particles only.

Data of the CMS+TOTEM experiment [18] at $\sqrt{s}=$ $8 \mathrm{TeV}$ are of especial interest. Figure 13 shows "NSDenhanced" $d n_{c h} / d \eta$ distributions for events selected at $\sqrt{s}=8 \mathrm{TeV}$ under the following requirements. Number of detected charged particles $n_{c h} \geq 1$ at $-6.5<\eta<$ -5.3 and $5.3<\eta<6.5$ (a); "more-forward" data for events with $n_{c h} \geq 1$ at $-6.5<\eta<-5.3$ or $5.3<\eta<$ 6.5 (b); "SD-enhanced" data derived for events with $n_{c h} \geq 1$ only at $-6.5<\eta<-5.3$ or only $5.3<\eta<6.5$ (lower triangles) (c); "more-forward" data obtained with

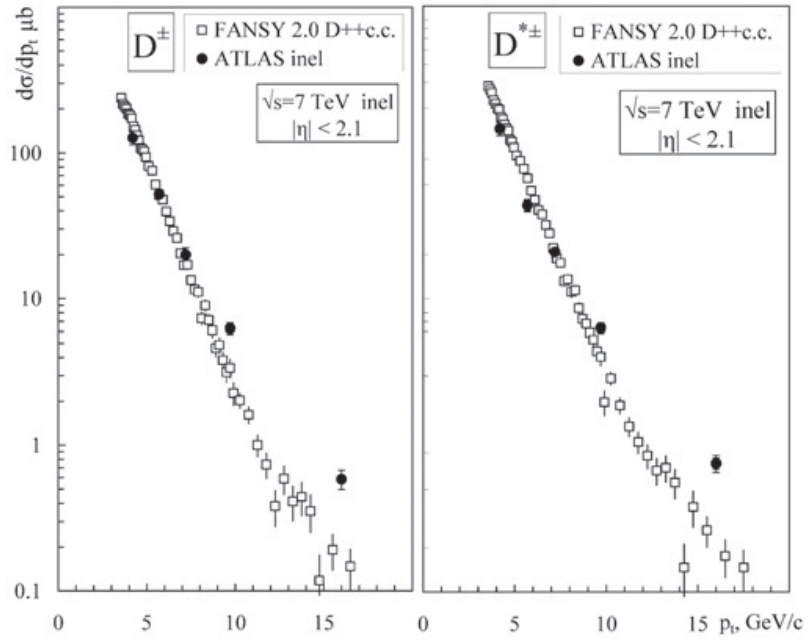

Figure 12. ATLAS $d \sigma / d p_{t}$ spectra of $\mathrm{D}^{ \pm}$(left) and $\mathrm{D}^{* \pm}$ (right) mesons at $|\eta|<2.1$ and $\sqrt{s}=7 \mathrm{TeV}$.

Table 2. $\mathrm{D}_{s}^{+} / \mathrm{D}^{+}, \mathrm{D}_{s}^{+} / \mathrm{D}^{0}$ ratios and ratio of vector mesons to prompt vector+pseudoscalar ones, $P_{V}$, at $|\eta|<0.5$. ALICE data include statistical and systematical errors. FANSY 2.0 results include statistical errors.

\begin{tabular}{cccc}
\hline Particles & $\begin{array}{c}p_{t}, \\
\mathrm{GeV} / \mathrm{c}\end{array}$ & ALICE & FANSY 2.0 \\
\hline $\mathrm{D}_{s}^{+} / \mathrm{D}^{+}$ & $2-12$ & $0.36 \pm 0.11 \pm 0.12$ & $0.35 \pm 0.01$ \\
$\mathrm{D}_{s}^{+} / \mathrm{D}^{0}$ & $2-12$ & $0.20 \pm 0.05 \pm 0.06$ & $0.15 \pm 0.01$ \\
$P_{V}=\mathrm{D}^{*+} /$ & & & \\
$\left(\mathrm{D}^{*+}+\mathrm{D}^{+}\right)$ & $1-24$ & $0.59 \pm 0.06 \pm 0.08$ & $0.55 \pm 0.01$ \\
\hline
\end{tabular}

displaced interaction points for events with $n_{c h} \geq 1$ at $-7.0<\eta<-6.0$ or $3.7<\eta<4.8$ (upper squares)(d).

Figure 14 shows FANSY 2.0 and the LHCf "neutron" $\left(n, \bar{n}, \mathrm{~K}_{s}, L^{0}\right) d \sigma / d x_{F}$ spectrum at $\left.p_{t}<0.11 x_{F}\right)$ at $\Delta \phi=360^{\circ}$ and $\sqrt{s}=7 \mathrm{TeV}$. Figure 15 shows LHCf and simulated "neutron" $d \sigma / d E$ energy spectra at $8.99<\eta<$ 9.2 (top) and at $\eta>10.76$ (bottom).

Figure 16 shows FANSY 2.0 and LHCf $\gamma$-ray $n_{\gamma} / N_{\text {inel }}$ energy spectra in the following pseudorapidity and azimuthal-angle ranges [2], $8.81<\eta<8.89, \Delta \phi=$ $20^{\circ}$ (top) and $\eta>10.94, \Delta \phi=360^{\circ}$ (bottom).

\section{Coplanar particle generation}

\subsection{General view}

A tendency to a coplanarity of the most energetic cores of $\gamma$-ray-hadron families observed was first found by the Pamir Collaboration [19-23] and confirmed later in [24-27]. The probability $W_{\text {tot }}^{\text {fluct }}$ for the total set of these experimental results to be produced by cascade fluctuations is much lower than $10^{-10}[1,28]$. This result illustrates that strong "forward-physics" interactions at superhigh energies are not well-described with the quarkgluon string model (QGSM) concept.

The phenomenon is related to hadron-nucleus interactions at $E_{0} \gtrsim 10^{16} \mathrm{eV}(\sqrt{s} \gtrsim 4 \mathrm{TeV})$ [29], characterized by a large cross section (comparable with $\sigma_{\text {inel }}^{p p}$ ) and was initially interpreted as a manifestation of large transverse momenta of the most energetic fragmentationrange particles $\left(X_{L a b}=E / E_{0} \gtrsim 0.1\right)$ [28]. 

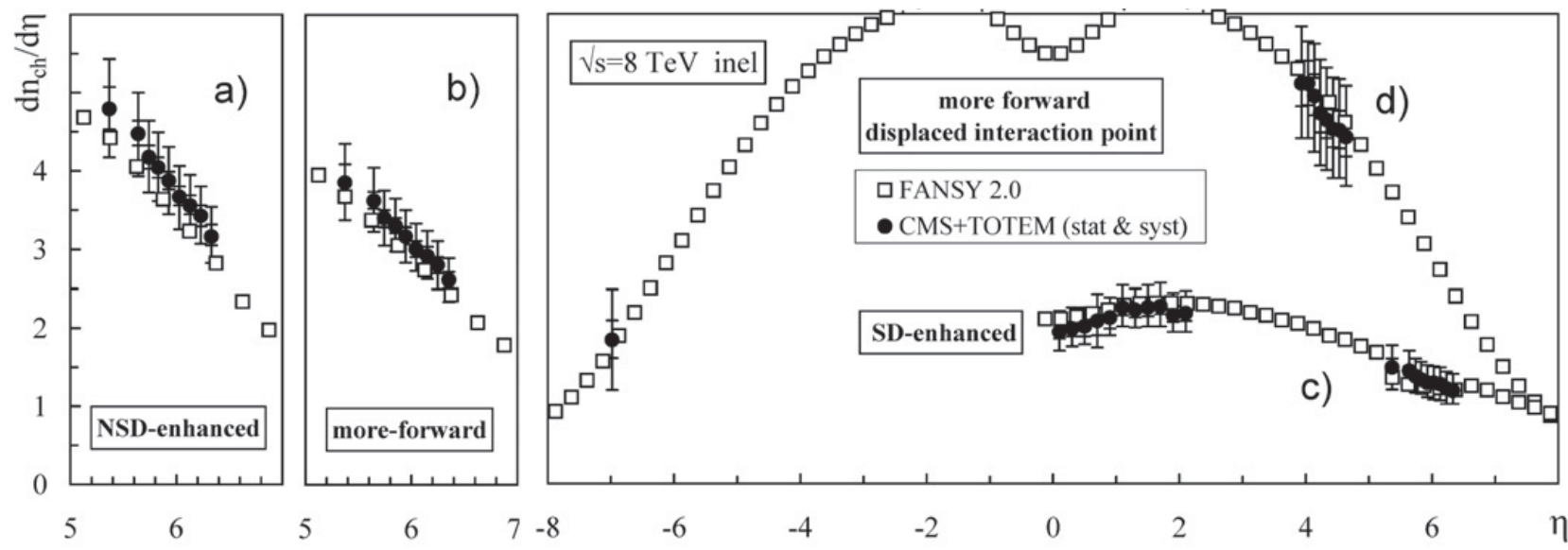

Figure 13. CMS+TOTEM and FANSY 2.0's $d n_{c h} / d \eta$ data for events selected at $\sqrt{s}=8 \mathrm{TeV}$ under the following requirements. "NSD-enhanced" data: $n_{c h} \geq 1$ in the ranges $-6.5<\eta<-5.3$ and $5.3<\eta<6.5$ (a); "more-forward" data: $n_{c h} \geq 1$ in the ranges $-6.5<\eta<-5.3$ or $5.3<\eta<6.5$ (b); "SD-enhanced" data (lower triangles): $n_{c h} \geq 1$ only in the ranges $-6.5<\eta<-5.3$ or only $5.3<\eta<6.5$ (c); "more-forward" data (upper squares) derived with displaced interaction points: $n_{c h} \geq 1$ in the ranges $-7.0<\eta<$ -6.0 or $3.7<\eta<4.8(\mathrm{~d})$.

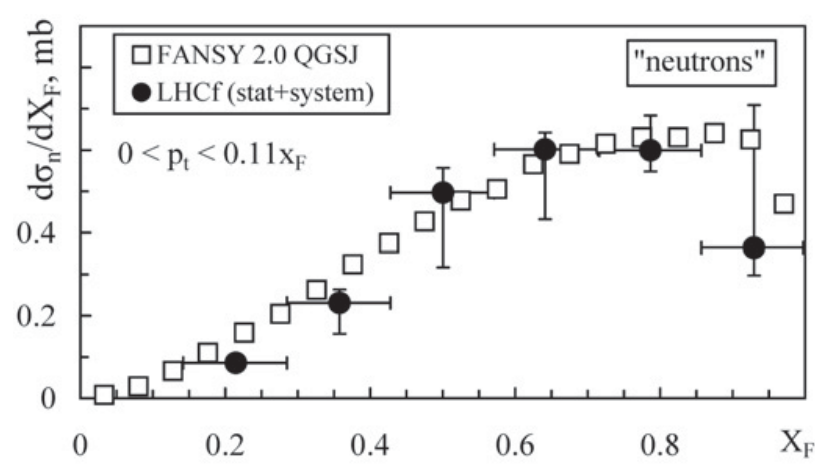

Figure 14. FANSY 2.0 and LHCf "neutron" $d \sigma / d x_{F}$ spectrum at $p_{t}<0.11 x_{F}$.

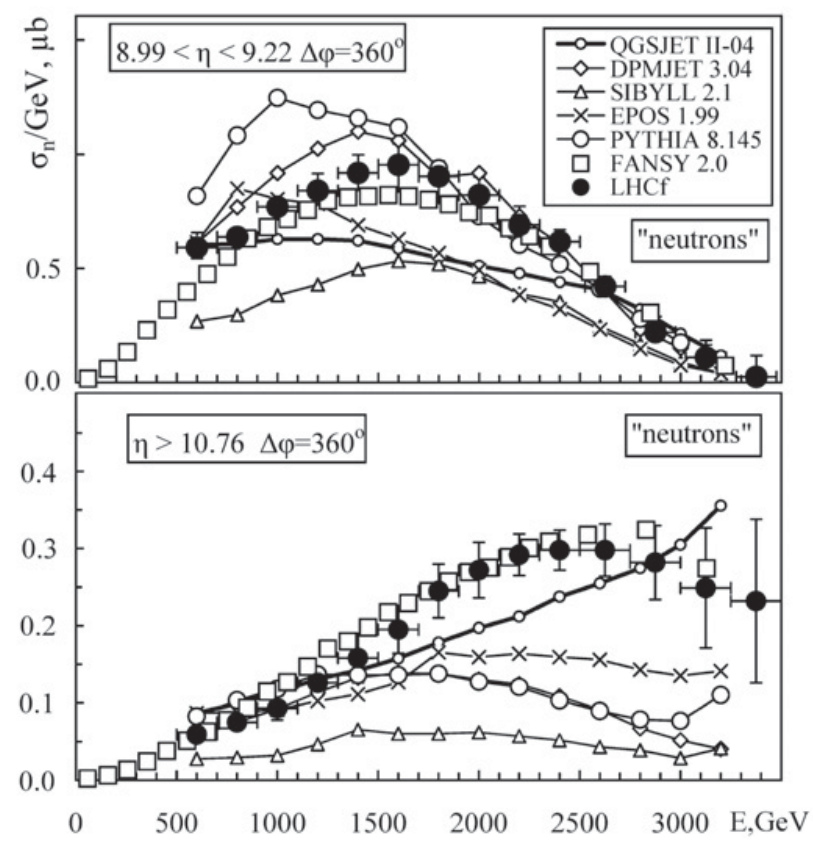

Figure 15. FANSY 2.0 and LHCf "neutron" $d \sigma / d E$ energy spectrum at $8.99<\eta<9.2$ (top) and at $\eta>10.76$ (bottom), $\Delta \phi=360^{\circ}$ and $\sqrt{s}=7 \mathrm{TeV}$.

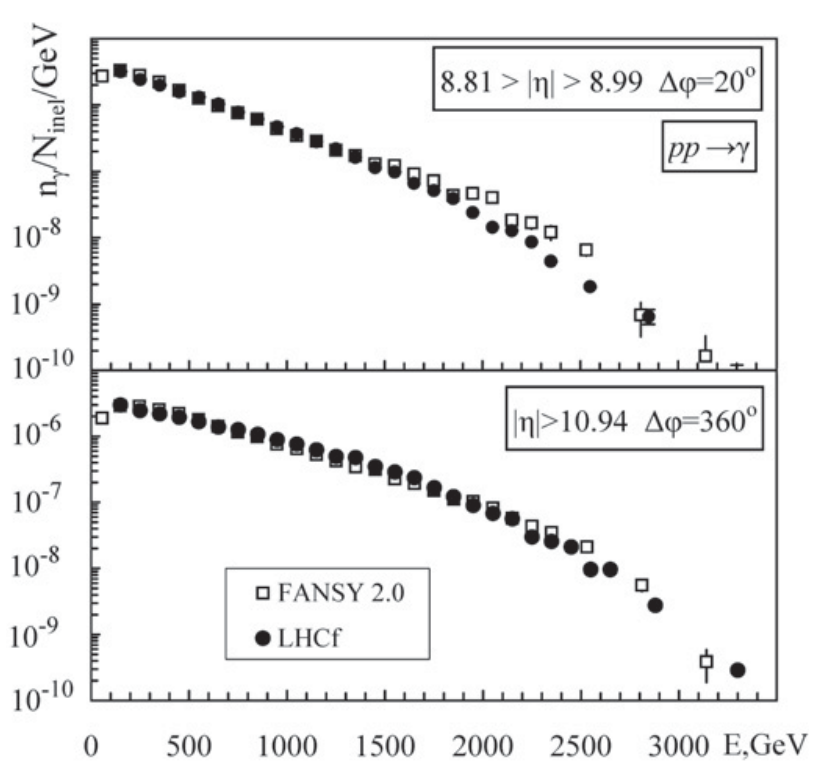

Figure 16. LHCf data and FANSY 2.0 results on $\gamma$-ray $n_{\gamma} / N_{\text {inel }} / \mathrm{GeV}$ energy spectra at $8.81<\eta<8.89, \Delta \phi=20^{\circ}$ (top) and $\eta>10.94, \Delta \phi=360^{\circ}$ (bottom).

This phenomenon could be reproduced in the framework of two concepts as a result of a) conservation of the angular momentum of a relativistic fast-rotating quark-gluon string (QGS) stretched between colliding hadrons [31]; b) semihard double diffraction (SHDID) dissociation and appearance of coplanarity as a result of QGS tension inside the diffraction cluster between a semihardly scattered constituent quark and other spectator quarks of the projectile hadron and its following rupture [32] with a lower multiplicity and higher average energy of particles; c) the most extraordinary explanation assumes that this phenomenon could be described within the recently proposed hypothesis of "crystal world", with latticed and anisotropic spatial dimensions and decrease of dimension number with increasing energy [33].

In this work, only the first approach is considered. 


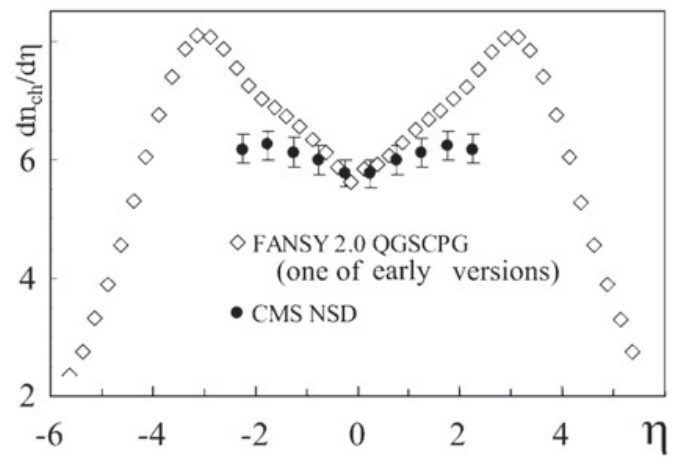

Figure 17. Peaks in $d \sigma / d \eta$ distribution originated by growth of transverse momenta of most energetic particles in primaryconcept CPG versions.
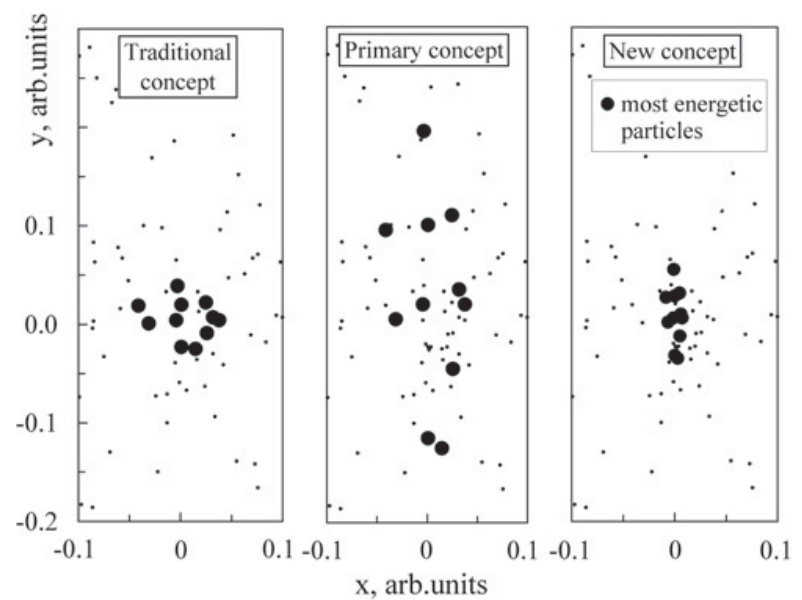

Figure 18. Tracks of particles on the target plane in cases of traditional interaction (left), coplanar interaction within the primary concept of increased $p_{t}$ (middle), coplanar interaction within the new concept of traditional $p_{t}$ (right). Most energetic particles are shown with large filled circles.

To study this problem, FANSY 2.0 QGSCPG version is designed [34], which simulates both traditional and coplanar particle generation. More details of comparison of LHC data with results of simulation with the abovedescribed basic FANSY 2.0 QGSJ version are given in [34].

\subsection{Coplanarity concepts}

Simulation using tentative FANSY 2.0 QGSCPG versions demonstrated the following fundamental problem.

The originally exploited concept qualitatively explains the observed coplanarity of momenta of most energetic particles with assuming their high transverse momenta in a coplanarity plane. However, in this case a significant $p_{t}$ growth suppresses $d \sigma / d y$ and $d \sigma / d \eta$ distributions of hadrons at highest $|y|$ and $|\eta|$ values and creates robust peaks at $2 \lesssim|\eta| \lesssim 4$ which are contrary to LHC data (Fig. 17). This was an unsolvable problem for all the QGSCPG versions based on the primary coplanarity concept. However, the coplanarity is observed in cosmicray experiments. Is it possible to reconcile this result with the LHC data?

Simulation has shown that a general agreement of LHC data and idea of coplanar generation becomes real

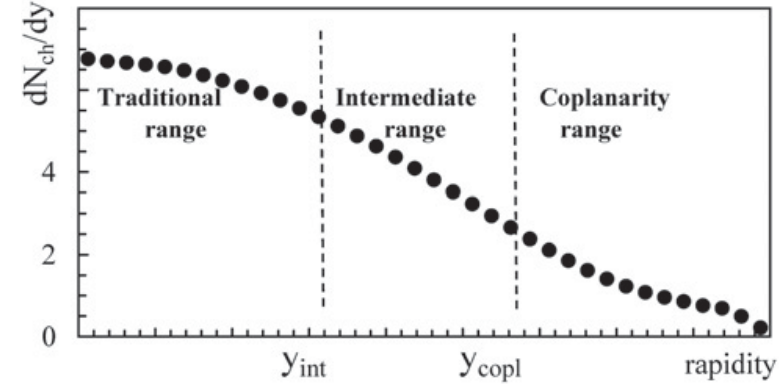

Figure 19. Qualitative dependence of the traditional, intermediate and coplanarity ranges on rapidity.

only using a new concept of coplanarity origin, namely, some decrease of particle's transverse momenta directed normally to the coplanarity plane takes place so that the absolute $p_{t}$ values do no change.

Figure 18 shows three examples of tracks of particles, generated in the same imaginary interaction, on a target plane, placed at some distance from the interaction point, in the cases of traditional QGSM-like interaction (left), primary-concept coplanar interaction with increased $p_{t}$ (middle), and new-concept coplanar interaction with traditional $p_{t}$ (right). Most energetic particles are shown with large black circles. The geometric scale is given in arbitrary units.

\subsection{Coplanarity simulation}

All simulated interaction characteristics (excluding azimuthal ones) simulated with FANSY 2.0 QGSJ and QGSCPG versions, are similar. The QGSJ and QGSCPG versions merge smoothly at $\sqrt{s} \lesssim 2 \mathrm{TeV}$.

In the QGSCPG version all characteristics of particles are primarily simulated with the traditional way. If the summary energy of secondary particles is higher than a fixed value, transversal momenta of high-rapidity particles in the coplanarity range $\left(|y|>y_{\text {copl }}\right)$, the algorithm turns $\vec{p}_{t}$ of each such particles towards the coplanarity plane. This plane is determined by the momenta of the interacting hadrons and $\vec{p}_{t}$ of leaders surviving after the collision. The trend of turning of transverse momenta of secondary particles to this plane is weakening at $y_{i n t}<|y|<y_{\text {copl }}$ (in the intermediate range) and disappears at $|y|<y_{\text {int }}$ ( the traditional range). Here $\left|y_{\text {max }}\right|=\sqrt{s} / 2 / m, y_{\text {copl }} \approx y_{\text {max }}-$ $\Delta y \approx 5-6, \quad y_{\text {int }} \approx 2-3, \quad \Delta y=3-5, m$ is particle mass.

Figure 19 shows a qualitative dependence of the traditional, intermediate and coplanarity ranges on rapidity.

\subsection{On the search for coplanarity at the LHC}

The CASTOR experiment seems to be promising in studying coplanarity (at least, in the framework of FANSY 2.0). Figure 20 shows a simplified CASTOR's cross section scheme and an example of detection of one coplanar interaction. The detector consists of 16 segments and is divided in the middle by a vertical slit. Particles are considered to be detected if their pseudorapidity values are in the range of $5.3<\eta<6.5$ and they do not fall into the vertical slit. Black circles in Fig. 20 show tracks of 


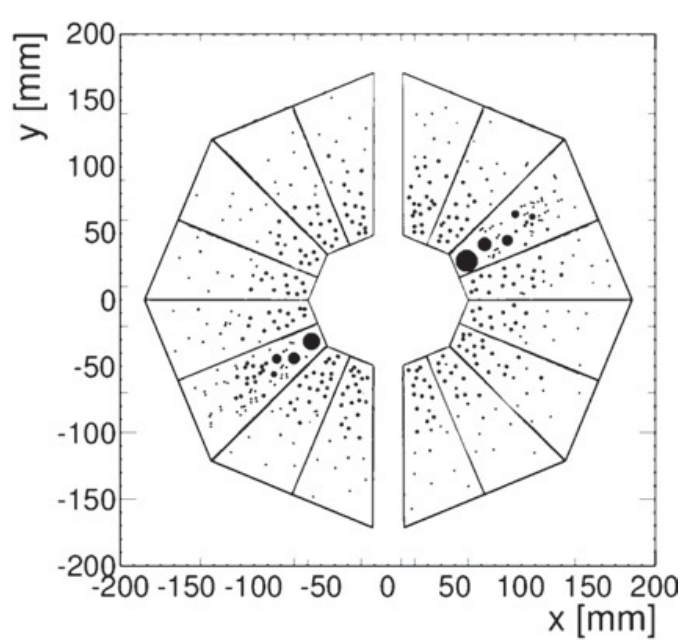

Figure 20. CASTOR's simplified cross section scheme. Black circles show tracks of particles. High-energy particles shown with large circles demonstrate a tendency to coplanarity.

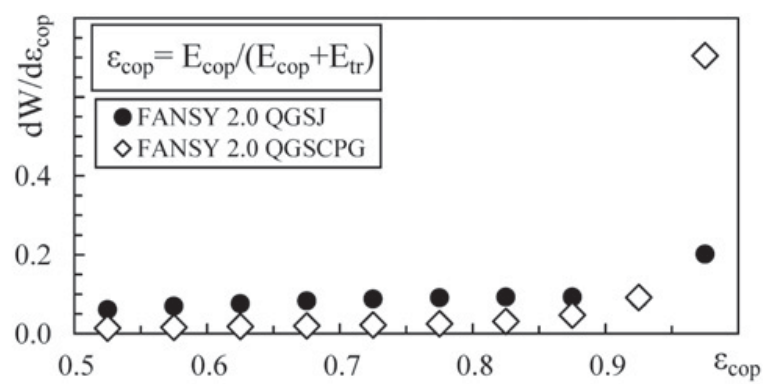

Figure 21. CASTOR's $\varepsilon_{c o p}$ distributions for FANSY 2.0 QGSJ and QGSCPG at $\sqrt{s}=7 \mathrm{TeV}$.

particles. The larger the circle size, the higher the energy of the particle. High-energy particles show a tendency to some coplanarity. Low-energy particles form a more or less azimuthally symmetric halo.

To analyze events, energy values "measured" in each of the segments, $E_{i}\left(E_{i} \geq 100 \mathrm{GeV}, i\right.$ is the number of a segment), are used. Events with total energy $\sum E_{i} \geq$ $1 \mathrm{TeV}$ "measured" in two or more segments are only analyzed. The first number is assigned to a segment with a maximum release of energy, $E_{\text {max }}$. Here and below, $E_{1}=$ $E_{\text {max }}, E_{\text {cop }}=E_{1}+E_{9} ; E_{t r}=E_{5}+E_{13}$, i.e., it is the energy measured in 9 th and 13 th segments, perpendicular to the first segment. A simple parameter is applied, namely, $\varepsilon_{c o p}=E_{c o p} /\left(E_{c o p}+E_{t r}\right)$, which characterizes the event coplanarity degree. If $\varepsilon_{c o p}=1$, the degree of event coplanarity is maximum.

Figure 21 shows $\varepsilon_{\text {cop }}$ distributions for FANSY 2.0 QGSJ and QGSCPG versions at $\sqrt{s}=7 \mathrm{TeV}$. Difference between model predictions becomes very large at $\varepsilon_{\text {cop }} \rightarrow 1$.

\section{Conclusion}

The FANSY 2.0 Monte Carlo code is designed to study superhigh-energy cosmic-ray "forward physics" interactions and includes traditional QGSJ QGSM-based version as well as a $Q G S C P G$ one which realizes a coplanar particle generation (CPG).

CPG process simulated with FANSY 2.0 QGSCPG does not contradict LHC data and could be tested in the CASTOR experiments. To reconcile experimental and simulated data, it is necessary to replace the primary concept of growth of transverse momenta of most energetic particles in the coplanarity plane with a new concept of reduction of transverse momenta directed normally to the coplanarity plane.

A version of the appearance of $\mathrm{CPG}$ processes in double-diffraction interactions only requires a separate consideration, as in this case the energy spectrum of secondary particles is more hard which is important in cosmic-ray experiments.

\section{References}

[1] R.A. Mukhamedshin, Eur. Phys. J. C 60, 345 (2009)

[2] O. Adriani, L. Bonechia, M. Bongia et al., Physics Letters B 703, 128-134 (2011)

[3] J. Kempa, R. Mukhamedshin, M. Tamada, J. Phys.: Conf. Ser. 409, 012109 (2013)

[4] R.A. Mukhamedshin, (to be sent to Eur.Phys.J.C )

[5] The ATLAS Collaboration, arXiv:1606.02625v2 [hep-ex]

[6] The CMS Collaboration, arXiv:1607.02033v1 [hep-ex]

[7] The ATLAS Collaboration, arXiv:1606.01133v1 [hep-ex]

[8] The ATLAS Collaboration, New J. Phys. 13, 053033 (2011)

[9] The CMS Collaboration, arXiv:1005.3299v2 [hep-ex]

[10] The LHCb Collaboration, Eur. Phys. J. C 72, 1947 (2011)

[11] The CMS Collaboration, J. High Energ. Phys. 05, 064 (2011)

[12] Phys. Lett. B 703 (2011) 267-273

[13] The ATLAS Collaboration. arXiv:1512.02913v1 [hep-ex]

[14] B. Abelev et al. (ALICE Collaboration). J. High Energ. Phys. 01, 128 (2012)

[15] The LHCb Collaboration, Nucl. Phys. B 871, 1 (2013)

[16] The LHCb collaboration. R.Aaij, C. Abelln Beteta et al., J. High Energ. Phys. 03, 159 (2016)

[17] The ALICE Collaboration, ArXiv: 1112.5 [hep-ex]

[18] arXiv: 1405.0722v1

[19] A. Borisov et al. (Pamir Collaboration), in Proc. 4th Int. Symp. on Very High Energy Cosmic Ray Interactions, Beijing 4 (1986)

[20] I.P. Ivanenko et al., JETP Lett. 50, 2125 (1992)

[21] V.V. Kopenkin et al., Phys. Rev. D 52, 2766 (1995)

[22] Pamir Collaboration, Preprint INP MSU no. 8967/144 (1989)

[23] Borisov A.S. et al., Nucl. Phys. B (Proc. Suppl.) 97, 118 (2001)

[24] L. Xue et al., in Proc. 26th Int. Cosmic Ray Conf., Salt Lake City 1, 127 (1999)

[25] A.V. Apanasenko et al., in Proc. 15th Int. Cosmic Ray Conf., Plovdiv 7, p. 220 (1977)

[26] A.K. Managadze et al., in Proc. 27th Int. Cosmic Ray Conf., Hamburg 1, p. 1426 (2001)

[27] J.N. Capdevielle, J.Phys. G 14, 503 (1988)

[28] R.A. Mukhamedshin, J. High Energy Phys. JHEP 05, 049 (2005) 
[29] A.S. Borisov et al., Nucl. Phys. B (Proc. Suppl.) 75A, 144 (1999)

[30] A.K. Managadze et al., in Proc. 29th Int. Cosmic Ray Conf., Pune 9, 81 (2005); A.K. Managadze et al., Physics of Atomic Nuclei 70(1), 184 (2007)

[31] T. Wibig, arXiv:0003230 [hep-ph]
[32] I.I. Royzen, Mod. Phys. Lett. A9(38), 3517 (1994)

[33] L.A. Anchordoqui, D.C. Dai, M. Fairbairnet et al., arXiv:1003.5914 [hep-ph]; L.A. Anchordoqui, D.C. Dai, H. Goldberg et al., arXiv:1012.1870v2 [hep-ph]

[34] R.A. Mukhamedshin (to be sent to Eur.Phys.J. C) 